\title{
Electron-Phonon Coupling and the Metallization of Solid Helium at Terapascal Pressures
}

\author{
Bartomeu Monserrat, ${ }^{1, *}$ N. D. Drummond, ${ }^{2}$ Chris J. Pickard,${ }^{3}$ and R. J. Needs ${ }^{1}$ \\ ${ }^{1}$ TCM Group, Cavendish Laboratory, University of Cambridge, J. J. Thomson Avenue, Cambridge CB3 OHE, United Kingdom \\ ${ }^{2}$ Department of Physics, Lancaster University, Lancaster LA1 4YB, United Kingdom \\ ${ }^{3}$ Department of Physics and Astronomy, University College London, Gower Street, London WC1E 6BT, United Kingdom
}

(Received 23 October 2013; published 7 February 2014)

\begin{abstract}
Solid $\mathrm{He}$ is studied in the pressure and temperature ranges 1-40 TPa and 0-10000 K using firstprinciples methods. Anharmonic vibrational properties are calculated within a self-consistent field framework, including the internal and free energies, density-pressure relation, stress tensor, thermal expansion, and the electron-phonon coupling renormalization of the electronic band gap. We find that an accurate description of electron-phonon coupling requires us to use a nonperturbative approach. The metallization pressure of $32.9 \mathrm{TPa}$ at $0 \mathrm{~K}$ is larger than found previously. The vibrational effects are large; for example, at $P=30 \mathrm{TPa}$ the band gap is increased by $2.8 \mathrm{eV}$ by electron-phonon coupling and a further $0.1 \mathrm{eV}$ by thermal expansion compared to the static value. The implications of the calculated metallization pressure for the cooling of white dwarfs are discussed.
\end{abstract}

PACS numbers: 63.20.Ry, 62.50.-p, 71.38.-k, 97.20.Rp

Helium $(\mathrm{He})$ is the second most abundant element in the Universe after hydrogen and an important component of stellar bodies such as giant gaseous planets, main-sequence stars, and white dwarf (WD) stars. The large first excitation energy of atomic $\mathrm{He}$ of $19.82 \mathrm{eV}$ leads to a high metallization pressure for the solid phase, of the order of tens of terapascals. Calculations [1,2] indicate that $\mathrm{He}$ remains solid at terapascal pressures up to temperatures of around $8000 \mathrm{~K}$ : solid $\mathrm{He}$ is therefore expected to occur in the outer layers of cool WDs.

The vast majority of stars in the Universe become WDs in their final stages of evolution, with the gravitational attraction towards the center being balanced by the electron degeneracy pressure of the high-density core. The lack of a continuous energy source means that WDs cool down until reaching thermodynamic equilibrium with their surroundings, eventually becoming black dwarfs. An understanding of the cooling process [3] is essential when calculating the ages of observed WDs, which are widely used within cosmochronology $[4,5]$ to date stellar clusters and galaxies, and hence to provide bounds on the age of the Universe.

The cores of WDs are largely isothermal due to the high thermal conductivity of degenerate electrons. Hence, the cooling rate is mainly determined by the outer layers, which are composed of hydrogen, $\mathrm{He}$, or a mixture of both. In this context the insulator-metal transition in solid $\mathrm{He}$ is central because energy transport from the degenerate core is dominated by electron transport through metallic $\mathrm{He}$ in the deeper layers, and by photon transport through insulating $\mathrm{He}$ in the outermost region [6].

Recent work has focused on studying the metallization pressure of $\mathrm{He}$ in the solid and fluid states [6-11]. In the solid state [6], static-lattice electronic structure calculations using both the diffusion Monte Carlo
(DMC) many-body technique $[12,13]$ and the $G W$ approximation of many-body perturbation theory [14] have shown that standard generalized gradient approximations [15] to density functional theory (DFT) [16,17] substantially underestimate band gaps. In the fluid state [7], the effects of electron-phonon coupling lead to a strong temperature dependence of the metallization pressure. The effects of temperature on the metallization transition in the solid state remain an open question.

Helium is the second lightest element and therefore the nuclear vibrational amplitudes are expected to be large and possibly to show anharmonicity. Recent advances in the treatment of anharmonicity from first principles make the incorporation of these effects feasible [18-23].

Both electron-phonon coupling and thermal expansion are important for determining band gaps in the solid state [24-26]. Studying the effects of electron-phonon coupling on the band gaps of solids from first principles has only recently become possible [22,27-30]. In this Letter, we combine first-principles calculations of anharmonicity, electron-phonon coupling, and thermal expansion to study the vibrational corrections to the thermal band gap of solid $\mathrm{He}$ and hence determine an accurate value for the temperature dependence of the metallization pressure.

We use the principal-axes approximation $[22,31]$ to the Born-Oppenheimer energy surface, considering independent phonon terms and pairwise phonon-phonon interactions. The resulting nuclear Schrödinger equation is solved within the vibrational self-consistent field (VSCF) framework [22,32] for the anharmonic vibrational energy $E_{\mathbf{S}}$ and wave function $\left|\Phi^{\mathbf{S}}(\mathbf{Q})\right\rangle$ in state $\mathbf{S}$, where $\mathbf{Q}$ is a collective phonon coordinate. We use second-order perturbation theory to go beyond the mean-field formulation. Phonon expectation values of a general operator $\hat{O}(\mathbf{Q})$ are calculated at finite temperature $T$ according to 


$$
\langle\hat{O}(\mathbf{Q})\rangle=\frac{1}{\mathcal{Z}} \sum_{\mathbf{S}}\left\langle\Phi^{\mathbf{S}}(\mathbf{Q})|\hat{O}(\mathbf{Q})| \Phi^{\mathbf{S}}(\mathbf{Q})\right\rangle e^{-E_{\mathbf{S}} / k_{B} T}
$$

where $\mathcal{Z}$ is the partition function and $k_{B}$ is Boltzmann's constant. This expectation value can be calculated by writing the operator $\hat{O}(\mathbf{Q})$ within a principal-axes representation like that for the energy [22], or nonperturbatively by sampling phase space according to the nuclear density $[28,30]$. The first method is approximate, but the description is in terms of individual phonons, permitting direct access to the underlying physical processes. The second method can, in principle, lead to accurate numerical results, but the underlying physical mechanisms are obscured. We use a combination of both methods to obtain a full picture of the effects of electron-phonon coupling on the band gap of He. We note that using DFT to calculate electron-phonon corrections to band gaps is reasonable, as the usual bandgap underestimation cancels in the difference between the static gap and the phonon-renormalized gap [29].

We solve the electronic Schrödinger equation to map the Born-Oppenheimer energy surface within plane-wave DFT $[16,17]$ using the CASTEP code [33]. We use ultrasoft pseudopotentials [34] with core radii of $0.212 \AA$, which require an energy cutoff of $2800 \mathrm{eV}$, and Monkhorst-Pack [35] k-point grids of spacing $2 \pi \times 0.04 \AA^{-1}$. These parameters lead to energy differences converged to within $10^{-4} \mathrm{eV}$ per atom and stresses to within $10^{-1} \mathrm{GPa}$. All calculations are performed with the Perdew-BurkeErnzerhof [15] generalized gradient approximation functional. We solve the vibrational Schrödinger equation within the VSCF formalism by expanding the wave function in a basis of simple harmonic oscillator eigenstates. This basis is defined from a quadratic fit to the Born-Oppenheimer energy surface calculated within the principal-axes approximation rather than from the harmonic approximation. Converged results are obtained using 100 simple harmonic oscillator states for each phonon degree of freedom.

The phase diagram of solid $\mathrm{He}$ at low pressures is well characterized, and the hexagonal closed-packed (hcp) structure with a $c / a$ ratio close to ideal is stable up to at least $58 \mathrm{GPa}$ [36]. It is usually assumed that He remains in the hcp phase up to high pressures and temperatures, but this does not seem to have been tested in detail. Furthermore, at the highest pressures it is expected to adopt the body-centered cubic (bcc) structure. We therefore perform searches at 10 and $20 \mathrm{TPa}$ using the $a b$ initio random structure searching (AIRSS) method $[37,38]$ to find low-enthalpy crystal structures of He with 12 or fewer atoms per cell. We calculate the harmonic vibrational free energy of the most competitive phases in the temperature range $0-10000 \mathrm{~K}$, finding several phases that are lower in free energy than the face-centered cubic (fcc) and bcc phases, but none of them were more stable than hcp. In Fig. 1 we show the static-lattice enthalpy $\Delta H_{\text {hcp }}$ of the

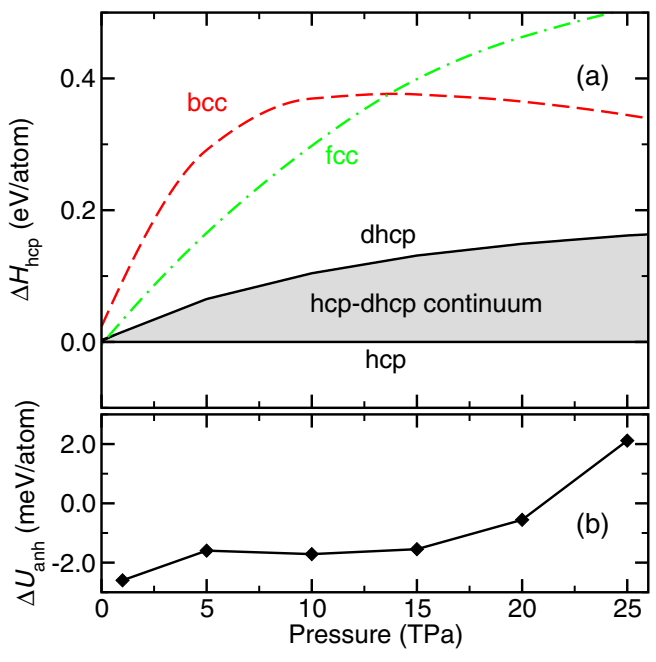

FIG. 1 (color online). (a) Enthalpy $\Delta H_{\text {hcp }}$ with respect to the hcp phase of the closed-packed phases described at the static DFT level. (b) Anharmonic energy correction $\Delta U_{\text {anh }}$ to the harmonic energy of the hcp phase at zero temperature.

closed-packed phases with respect to the hcp phase. The hcp and double hexagonal closed-packed (dhcp) structures are the two end members of a series of structures which differ in the stacking of layers, and whose energies per layer vary continuously with the fraction of stacking faults present.

We have also calculated the anharmonic free energy $\Delta F_{\text {anh }}$ of hcp He (see Fig. 1). At $10 \mathrm{TPa}$ and zero temperature, for example, the harmonic energy is $686.9 \mathrm{mev} /$ atom and the anharmonic correction from the independent phonon term is $-1.7 \mathrm{meV} /$ atom, which is further renormalized by $+1.1 \mathrm{meV} /$ atom due to the twobody phonon term. At $T=5000 \mathrm{~K}$, the harmonic free energy is $75.0 \mathrm{meV} /$ atom, and the anharmonic correction is $-5.2 \mathrm{meV} /$ atom. At $25 \mathrm{TPa}$ and zero temperature the harmonic energy is $903.0 \mathrm{meV} /$ atom and the anharmonic correction is only $2.1 \mathrm{meV} /$ atom, whereas at $T=5000 \mathrm{~K}$, the harmonic free energy is $466.6 \mathrm{meV} /$ atom and the anharmonic correction is $6.3 \mathrm{meV} /$ atom. Second-order perturbation theory does not change these results within the reported precision, demonstrating the accuracy of the mean-field approximation. These anharmonic corrections, albeit larger than in heavier systems such as diamond [22], remain remarkably small and have no discernible effect on the relative stability of the phases considered. Furthermore, the ratio of the anharmonic to quasiharmonic vibrational free energy decreases with increasing pressure, suggesting that anharmonicity becomes less important for the energetics of He at high pressures.

The band structure and density of states of hcp He at $10 \mathrm{TPa}$ are shown in Fig. 2. The occupied density of states is very similar to that of a free electron gas at the same density, only deviating from it in the gap region. The valence-band maximum (VBM) is located along the line joining the $\Gamma$ and $M$ points, while the conduction-band 


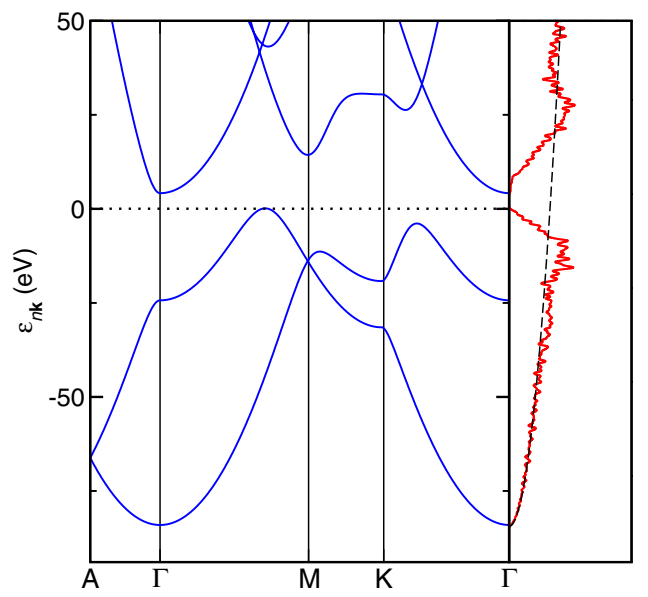

FIG. 2 (color online). Electronic band structure (blue, on lefthand side of figure) and density of states (red, on right-hand side of figure) of hcp $\mathrm{He}$ at $10 \mathrm{TPa}$. The dashed black line is the density of states of a free electron gas of the same density as He.

minimum $(\mathrm{CBM})$ is located at the $\Gamma$ point, as observed experimentally at lower pressures [39]. We have evaluated Eq. (1), with $\hat{O}$ being the separation of the VBM and CBM, both within a principal-axes representation and nonperturbatively [40]. Figure 3 shows the pressure dependence of the zero-point (ZP) electron-phonon correction to the thermal band gap of hcp He calculated nonperturbatively. A second calculation of the same quantity with a principalaxes representation of $E_{\mathrm{g}}(\mathbf{Q})$ gives information about the underlying physical mechanisms. The out-of-plane modes of the hcp lattice couple strongly to the electronic bands, which opens the band gap. This coupling increases with pressure, leading to the behavior shown in Fig. 3. This can be further understood by analyzing the Kohn-Sham eigenstates corresponding to the VBM and the CBM (Fig. 3). At lower pressure these eigenstates are localized around the atomic sites, but as pressure increases they delocalize in the interplane direction, hence increasing the coupling with the out-of-plane modes.

We emphasize that the nonperturbative calculation of the renormalization of the band gap due to electron-phonon coupling leads to quantitatively different results to the perturbative approach. Terms beyond lowest-order perturbation theory are crucial for calculating the band-gap correction [41]. The magnitude of the band-gap renormalization is amongst the largest reported, similar only to that found in high-pressure solid hydrogen [42].

To investigate thermal expansion we have calculated the vibrational stress tensor at finite temperatures using the formalism of Ref. [22]. The vibrational stress tensor is diagonal, with similar in-plane and out-of-plane stresses, leading to an isotropic volume expansion. Figure 4 shows the density-pressure relation at five different temperatures, including vibrational effects. Both $\mathrm{ZP}$ and finitetemperature expansions are more significant at lower

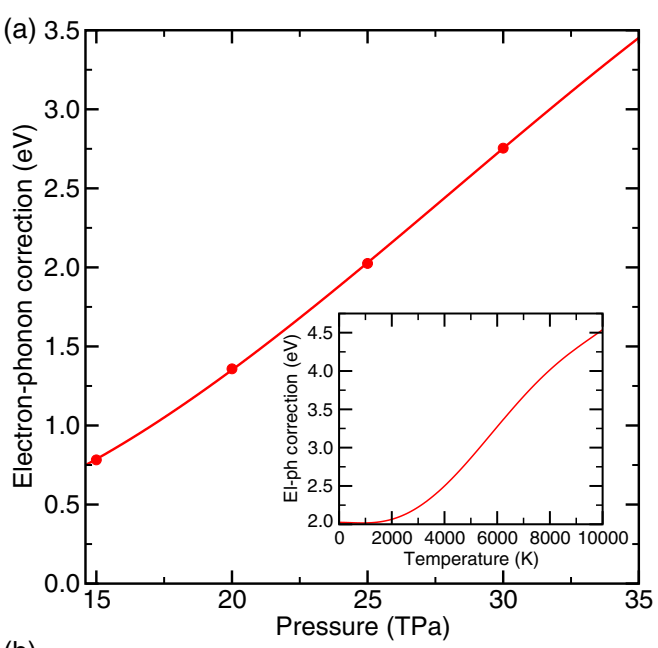

(b)

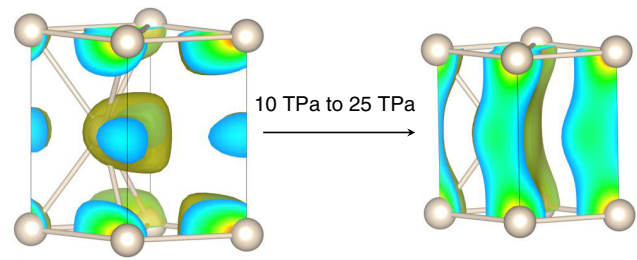

FIG. 3 (color online). (a) ZP electron-phonon correction to the thermal band gap of hcp solid He as a function of pressure. The inset shows the temperature dependence of the electron-phonon correction at a pressure of $P=25 \mathrm{TPa}$. (b) Squared Kohn-Sham eigenstate corresponding to the CBM at 10 and $25 \mathrm{TPa}$, using a red-green-blue color scale with red corresponding to high density and blue to low density. We have removed the regions of lowest density for clarity.

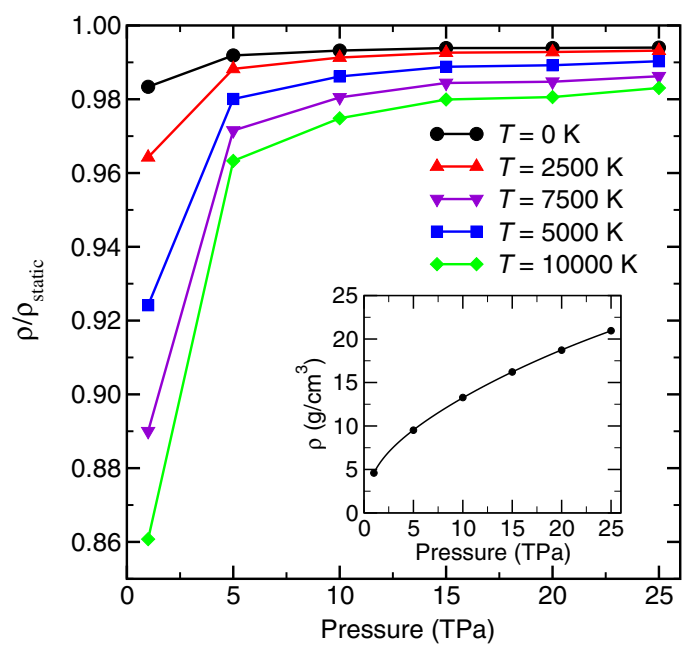

FIG. 4 (color online). Density-pressure-temperature phase diagram of solid hcp He. At a given external pressure, the density $\rho$ is renormalized with respect to the static-lattice density $\rho_{\text {static }}$ at that pressure. The inset shows the density-pressure relation at $T=0 \mathrm{~K}$, including the effects of $\mathrm{ZP}$ motion. 


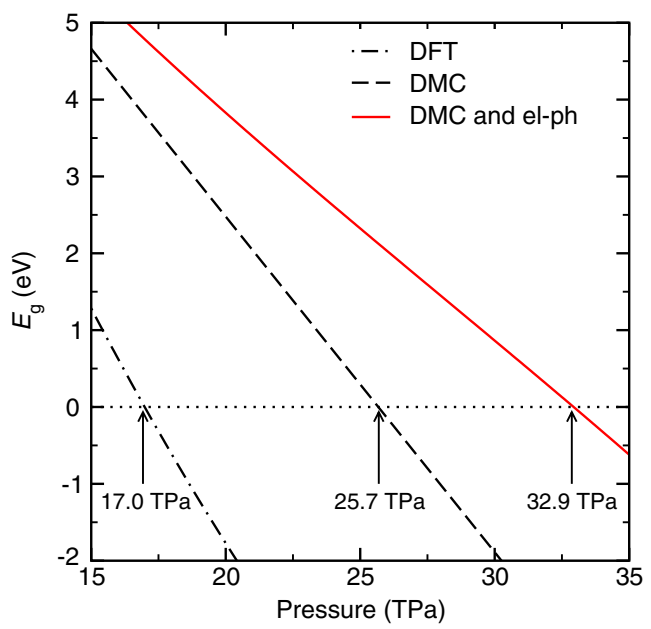

FIG. 5 (color online). Thermal band gap $E_{\mathrm{g}}$ of solid hcp He as a function of pressure, calculated by evaluating the difference between the CBM and the VBM (even when the difference is negative). The static gap results correspond to DFT calculations (dash-dotted black line) and DMC calculations (dashed black line) taken from Ref. [6]. The band gap, including the effects of electron-phonon coupling and thermal expansion added to the DMC results, is shown at $T=0 \mathrm{~K}$ (red solid line).

pressures. At $P=25 \mathrm{TPa}$, the $\mathrm{ZP}$ correction to the volume at $T=0 \mathrm{~K}$ opens the gap by $0.105 \mathrm{eV}$, and the thermal expansion at $T=5000 \mathrm{~K}$ opens it by $0.216 \mathrm{eV}$

Combining the effects of electron-phonon coupling and thermal expansion, we calculate the pressure dependence of the thermal band gap of solid He near metallization, as shown in Fig. 5. The static-lattice DMC results are those reported in Ref. [6], which agree with $G W$ results. Including electron-phonon coupling and $\mathrm{ZP}$ expansion at zero temperature leads to metallization at $32.9 \mathrm{TPa}$, significantly higher than the static-lattice value.

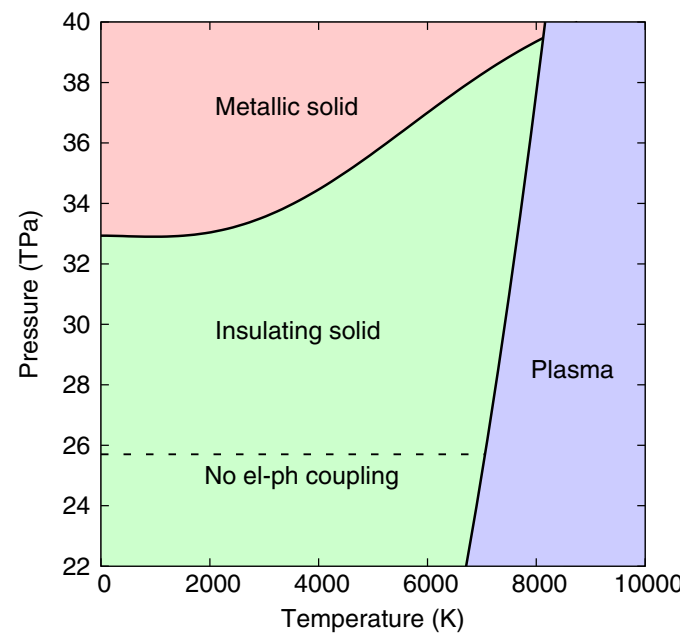

FIG. 6 (color online). Phase diagram of $\mathrm{He}$ at the pressuretemperature range relevant for cool WDs. The black dashed line is the insulator-metal transition predicted in Ref. [6]. The solidplasma transition is taken from Ref. [2].
The vibrational band-gap renormalization near the metallization pressure is about $3.0-4.0 \mathrm{eV}$ for temperatures in the range $0-5000 \mathrm{~K}$ This represents a quantitative change in the static-lattice value of the same order of magnitude as the electron-correlation correction to DFT [6]. Furthermore, our results are the first to describe the temperature dependence of the transition. The authors of Ref. [6] considered the effects of temperature on the electronic band gap within path integral Monte Carlo [43], and concluded that they are negligible. We have come to a significantly different conclusion on this issue, but there is insufficient information in Ref. [6] to allow a more detailed comparison. Combining our data with those from Ref. [2], we construct the phase diagram of $\mathrm{He}$ at the pressures and temperatures relevant for cool WDs shown in Fig. 6.

In conclusion, we have presented first-principles calculations of the phase stability, electron-phonon coupling, and thermal expansion of solid He over a range of pressures and temperatures. We have shown that the thermodynamically stable phase of solid He is hcp for the pressure range 1-30 $\mathrm{TPa}$ and temperature range $0-10000 \mathrm{~K}$ including anharmonic energies at the mean-field level. The secondorder perturbation theory used to go beyond the VSCF approximation gives a negligible correction, suggesting that the mean-field energy is accurate. The effects of electron-phonon coupling on the band gap are substantial and several times larger than the effects of thermal expansion. A perturbative approach is inaccurate here and a nonperturbative scheme, as we have used, is required. We have determined the metal-insulator transition of solid He to be at $32.9 \mathrm{TPa}$ at $T=0 \mathrm{~K}$ which may be compared with the value $25.7 \mathrm{TPa}$ obtained in Ref. [6] at the staticlattice level.

The increase in the metallization pressure of solid $\mathrm{He}$, including the effects of electron-phonon coupling and thermal expansion, implies that the interiors of WDs have a thinner metallic He layer than predicted by a purely electronic treatment, and a correspondingly thicker insulating layer where heat transport is dominated by photons. The temperature dependence of the metallization pressure indicates that, as WDs cool, the thickness of the metallic layer increases at the expense of the insulating layer. These results suggest that white dwarf stars may be older than previously thought.

Financial support was provided by the Engineering and Physical Sciences Research Council (U.K.). The calculations were performed on the Cambridge High Performance Computing Service facility.

*bm418@cam.ac.uk

[1] D. A. Young, A. K. McMahan, and M. Ross, Phys. Rev. B 24, 5119 (1981).

[2] A. Förster, T. Kahlbaum, and W. Ebeling, Laser Part. Beams 10, 253 (1992). 
[3] F. D’Antona and I. Mazzitelli, Annu. Rev. Astron. Astrophys. 28, 139 (1990).

[4] D. E. Winget, C. J. Hansen, J. Liebert, H. M. van Horn, G. Fontaine, R. E. Nather, S. O. Kepler, and D. Q. Lamb, Astrophys. J. Lett. 315, L77 (1987).

[5] T. D. Oswalt, J. A. Smith, M. A. Wood, and P. Hintzen, Nature (London) 382, 692 (1996).

[6] S. A. Khairallah and B. Militzer, Phys. Rev. Lett. 101, 106407 (2008).

[7] L. Stixrude and R. Jeanloz, Proc. Natl. Acad. Sci. U.S.A. 105, 11071 (2008).

[8] P. M. Kowalski, S. Mazevet, D. Saumon, and M. Challacombe, Phys. Rev. B 76, 075112 (2007).

[9] P. M. Celliers, P. Loubeyre, J. H. Eggert, S. Brygoo, R. S. McWilliams, D. G. Hicks, T. R. Boehly, R. Jeanloz, and G. W. Collins, Phys. Rev. Lett. 104, 184503 (2010).

[10] F. Soubiran, S. Mazevet, C. Winisdoerffer, and G. Chabrier, Phys. Rev. B 86, 115102 (2012).

[11] J. M. McMahon, M. A. Morales, C. Pierleoni, and D. M. Ceperley, Rev. Mod. Phys. 84, 1607 (2012).

[12] D. M. Ceperley and B. J. Alder, Phys. Rev. Lett. 45, 566 (1980).

[13] W. M. C. Foulkes, L. Mitas, R. J. Needs, and G. Rajagopal, Rev. Mod. Phys. 73, 33 (2001).

[14] F. Aryasetiawan and O. Gunnarsson, Rep. Prog. Phys. 61, 237 (1998).

[15] J. P. Perdew, K. Burke, and M. Ernzerhof, Phys. Rev. Lett. 77, 3865 (1996).

[16] P. Hohenberg and W. Kohn, Phys. Rev. 136, B864 (1964).

[17] W. Kohn and L. J. Sham, Phys. Rev. 140, A1133 (1965).

[18] P. Souvatzis, O. Eriksson, M. I. Katsnelson, and S. P. Rudin, Phys. Rev. Lett. 100, 095901 (2008).

[19] I. Errea, B. Rousseau, and A. Bergara, Phys. Rev. Lett. 106, 165501 (2011).

[20] O. Hellman, I. A. Abrikosov, and S. I. Simak, Phys. Rev. B 84, 180301 (2011).

[21] N. Antolin, O. D. Restrepo, and W. Windl, Phys. Rev. B 86, 054119 (2012).

[22] B. Monserrat, N. D. Drummond, and R. J. Needs, Phys. Rev. B 87, 144302 (2013).

[23] I. Errea, M. Calandra, and F. Mauri, Phys. Rev. Lett. 111, 177002 (2013).
[24] M. Cardona and M. L. W. Thewalt, Rev. Mod. Phys. 77, 1173 (2005).

[25] P. B. Allen and V. Heine, J. Phys. C 9, 2305 (1976).

[26] P. B. Allen and M. Cardona, Phys. Rev. B 23, 1495 (1981).

[27] R. D. King-Smith, R. J. Needs, V. Heine, and M. J. Hodgson, Europhys. Lett. 10, 569 (1989).

[28] R. Ramírez, C. P. Herrero, and E. R. Hernández, Phys. Rev. B 73, 245202 (2006).

[29] F. Giustino, S. G. Louie, and M. L. Cohen, Phys. Rev. Lett. 105, 265501 (2010).

[30] C. E. Patrick and F. Giustino, Nat. Commun. 4, 2006 (2013).

[31] J. O. Jung and R. B. Gerber, J. Chem. Phys. 105, 10332 (1996).

[32] J. M. Bowman, J. Chem. Phys., 68, 608 (1978).

[33] S. J. Clark, M. D. Segall, C. J. Pickard, P. J. Hasnip, M. I. J. Probert, K. Refson, and M. C. Payne, Z. Kristallogr. 220, 567 (2005).

[34] D. Vanderbilt, Phys. Rev. B 41, 7892 (1990).

[35] H. J. Monkhorst and J. D. Pack, Phys. Rev. B, 13, 5188 (1976).

[36] P. Loubeyre, R. LeToullec, J. P. Pinceaux, H. K. Mao, J. Hu, and R. J. Hemley, Phys. Rev. Lett. 71, 2272 (1993).

[37] C. J. Pickard and R. J. Needs, Phys. Rev. Lett. 97, 045504 (2006).

[38] C. J. Pickard and R. J. Needs, J. Phys. Condens. Matter 23, 053201 (2011).

[39] H. K. Mao, E. L. Shirley, Y. Ding, P. Eng, Y. Q. Cai, P. Chow, Y. Xiao, J. Shu, R. J. Hemley, C. Kao, and W. L. Mao, Phys. Rev. Lett. 105, 186404 (2010).

[40] Supercells containing 54 atoms are used for all reported calculations. Tests with supercells containing up to 250 atoms show a convergence of the band-gap corrections within $0.2 \mathrm{eV}$, leading to an uncertainty of $0.4 \mathrm{TPa}$ in the metallization pressure. For the nonperturbative calculations, a total of 1000 sampling points have been used at each pressure and temperature, leading to statistical error bars smaller than the reported accuracy.

[41] B. Monserrat, G. J. Conduit, and R. J. Needs, arXiv:1308.3483.

[42] M. A. Morales, J. M. McMahon, C. Pierleoni, and D. M. Ceperley, Phys. Rev. B 87, 184107 (2013).

[43] D. M. Ceperley, Rev. Mod. Phys. 67, 279 (1995). 